\section{La figura de la Film Commission en la puesta en valor de los recursos audiovisuales técnicos y profesionales de un territorio en España}

The figure of the Film Commission in the put in value of the technical and professional audiovisual resources of a territory in Spain

Sarabia, Isabela; Sánchez, Josefina ${ }^{\mathrm{b}}$;

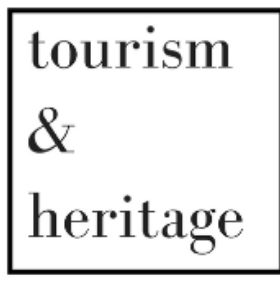

J O U R N A L
INFO DEL ARTÍCULO

Historial del artículo:

Recibido 23 julio2019

Aceptado 6 septiembre 2019

Publicado 25 septiembre 2019

\section{RESU M EN}

El interés en convertir un territorio en un escenario de rodaje de producciones audiovisuales cinematográficas, series televisivas o cualquier otro tipo de producto audiovisual, constituye el principal motor de la puesta en marcha de una Film Office o Film Commission en cualquier localidad, comarca, provincia o región.

La misión de una oficina de rodajes es poner de relieve, no sólo las bondades de los paisajes, monumentos, clima de un territorio o la capacidad de su gente de acoger equipos de rodaje (logística, predisposición de los ciudadanos), sino también dar visibilidad a los recursos técnicos y humanos audiovisuales con los que un equipo de rodaje llegado a una localidad podría contar en ese entorno.

Con esta investigación se pretende estudiar la figura de la Film Commission y su contribución a la dinamización de la producción en un territorio mediante la puesta en valor del sector audiovisual local empresas y técnicos especializados- como atractivo del territorio. producción audiovisual, recursos profesionales, promoción del territorio

\section{A B S T R A C T}

The interest in turning a territory into a setting for the filming of cinematographic audiovisual productions, television series or any other type of audiovisual product, constitutes the main engine of the start-up of a Film Office or Film Commission in any locality, region, province or region.

The mission of a filming office is to highlight, not only the benefits of landscapes, monuments, climate of a territory or the ability of its people to host filming equipment (logistics, predisposition of citizens), 
Keywords:

Film Commission, audiovisual production, professional resources, promotion of the territory but also to give visibility to the technical and human audiovisual resources with which a shooting team arrived in a locality could count on in that environment.

This research aims to study the figure of the Film Commission and its contribution to the dynamization of production in a territory through the enhancement of the local audiovisual sector -companies and specialized technicians- as an attractiveness of the territory.

\section{INTRODUCCIÓN}

La Association of Film Commissioners International (AFCI), órgano de máxima representatividad de la actividad de las oficinas fílmicas, y que reúne a más de 300 agencias de todo el mundo, las define como "... a specialized office under the authority of a government entity, or administrative office, with the purpose of promoting the region through the development of film, video, and multimedia production" (AFCI, 2019, s.p.)

La AFCI señala tres principales aspectos en la configuración de una Film Commission (FC). De un lado, se alude al hecho de que se trata de una agencia que provee de unos servicios especializados en materia audiovisual a unos clientes. Por lo tanto, su ámbito de actuación gira específicamente en torno a la producción de contenidos audiovisuales. Por otro lado, un aspecto esencial que define la figura de la Film Commission, y sobre lo que la AFCI llama la atención, es que se trata de un órgano vinculado a la administración pública. Es decir, es un servicio público, por lo tanto, esto implica que la oficina es fruto de una política pública en la que pueden intervenir diferentes departamentos y áreas de la administración. Respecto al hecho de que se trate de un servicio ofrecido en el seno de una entidad pública, según algunos autores no siempre es así, ya que en determinadas situaciones se abre la posibilidad a que la oficina tenga una ejecución privada. Pero, en cualquier caso, en lo que todas deben coincidir para ser consideradas como tales FCs es en su carácter de entidad sin ánimo de lucro, y en la gratuidad en la prestación de sus servicios (Ramahi, 2011; Figueira, Figueira y Monteiro, 2015).

El tercer aspecto que vertebra la definición de la AFCI es el que se refiere a la promoción del territorio a través del audiovisual. Esta función atribuida a esta modalidad de agencias genera beneficio en dos industrias, la turística y la audiovisual. Esta es una realidad que se ha esbozado desde el ámbito académico. En este sentido, autores como Palmi, Caputo y Turco señalan ese aspecto en los siguientes términos:

"FCs are traditionally considered territorial attraction agencies that act by making the territorial potentialities operate systematically, bringing cinema and audiovisual productions - and through them, promoting the territory as well. Therefore, on the one hand they aim to benefit from the production economically; on the other hand, they try to capitalize on the territory image promotion, thanks to the ability of audiovisual products to function as tourist attraction factors" (Palmi, Caputo $\mathrm{y}$ Turco, 2016, p. 57). 
El profesor Marcelo Martínez también insiste en esta virtud de las FCs, y cuando se refiere a su papel, alude tanto al impulso de la actividad audiovisual en el territorio, como a la explotación del retorno por la actividad audiovisual que una productora ha tenido en ese lugar. De modo que, para este autor, una oficina fílmica se ocupa tanto de los intereses de la industria audiovisual como los del turismo y la coordinación de las acciones conjuntas (Martínez, 2003). Reforzando el matiz de la rentabilidad turística de la actividad de la Film Commission, el investigador Eugeni Osácar apunta por su parte, a la virtual colaboración entre estas agencias y las Destination Management Organizations orientada a:

"Atraer grandes producciones a los escenarios naturales, captar nuevos segmentos de público, aumentar las visitas a lugares menos conocidos, posicionar el destino o crear nuevos productos turístico-culturales son algunas de las consecuencias de este nuevo marketing estratégico y operativo aplicado al turismo cinematográfico" (Osácar, 2016, p. 845-846).

Se pone, por lo tanto, de relieve que las dos industrias involucradas en la actividad de la Film Commission y beneficiadas por ella son tanto la turística como la audiovisual. Si bien el efecto positivo recae en ambos sectores, Marcelo Martínez advierte que, en ocasiones, este puede inclinarse más en un sector que en otro dependiendo, de un lado, del modelo de Film Commission de que se trate y, de otro, del ámbito o área de la institución pública en la que se ha desarrollado la iniciativa - cultura, turismo, fomento...- (Martínez, 2003).

En esta investigación el foco del trabajo se centra en la vinculación de la Film Commission con el sector audiovisual. De modo que la aproximación a la actividad de la Film Commission se realiza desde la perspectiva de la repercusión que esta puede generar en el sector audiovisual. Autores como Figueira, Figueira y Monteiro ponen el acento en este aspecto, y plantean la Film Commission como una entidad "... whose main objective is to promote the development of cinema, multimedia and audiovisual sectors in the region concerned" (Figueira, Figueira y Monteiro, 2015, p. 30).

En el marco de este enfoque -la Film Commission como instrumento de promoción de la industria audiovisual- cabe diferenciar dos perfiles de empresas y profesionales involucrados y virtualmente favorecidos por la actuación de la FC. De un lado, se encuentran aquellos que eligen un territorio como localización de su rodaje, y de otro, se identifica al tejido audiovisual local que acoge y da servicio a esa producción. De estos dos agentes beneficiados, los que se instalan en un territorio para desarrollar sus rodajes, y aquellos profesionales que se encuentran ya en él dispuestos a ser contratados, este trabajo se centra en el segundo, es decir, en el sector audiovisual local que atiende los rodajes. Por lo tanto, en esta investigación se hace foco en la relación de la FC con la industria audiovisual local.

Este trabajo parte de la hipótesis de que la FC es un instrumento de visibilidad del sector de la producción local y actúa, por lo tanto, como escaparate del activo de profesionales y empresas audiovisuales de su ámbito de cobertura.

Se aborda la FC como un agente al que, desde la perspectiva académica se le ha prestado muy poca atención como dinamizador de la industria audiovisual, pero que, sin embargo, se erige como uno de los dispositivos de apoyo al sector de la producción audiovisual de 
especial relevancia no sólo por su apoyo in situ a las empresas externas, sino, sobre todo, por su promoción de la industria local.

De modo que mediante este estudio se aspira, como principal objetivo, a poner en valor el comportamiento de una Film Commission en tanto que escaparate de los recursos técnicos audiovisuales de su territorio -profesionales y empresas de servicios audiovisuales-. Y en esta línea, se propone, de un lado, identificar el modo en el que las oficinas van construyendo y sumando tanto a técnicos y compañías en su oferta; conocer el modo en el que estos recursos son presentados; así como trazar el tipo de perfiles profesionales y empresariales que se ponen a disposición de las compañías de producción externas y observar cómo están organizados.

Para desarrollar estos objetivos se ha acudido a diferentes metodologías. Por una parte, se han revisado las webs de las 35 Film Commissions inscritas en la Spain Film Commission, con la finalidad de recabar toda la información referida a la oferta de profesionales y empresas audiovisuales y auxiliares locales, y analizar su presentación. La recogida de datos se ha sistematizado con la ayuda de una ficha de análisis en la que se han contemplado los siguientes ítems:

- Información sobre profesionales y empresas

- Modo de presentación de los datos

- Gestión de los datos y perfiles de profesionales y empresas locales en la FC

Con la intención de conocer cuáles son las especialidades profesionales y el tipo de empresas que se ofrecen en la página web, se ha realizado un estudio particular de la Andalucía Film Commission. Se trata de una entidad que se caracteriza por tener una larga trayectoria, así como una intensa actividad. En este trabajo se traza, a partir de los datos obtenidos del análisis de su página web, un mapa de profesionales y empresas visibilizados por la FC mediante una presentación apoyada en tablas de datos.

\section{DESARROLLO DE LAS FILM COMMISSIONS}

Las oficinas fílmicas tienen su origen en EE.UU. a mediados de los años 40 del siglo pasado como consecuencia del interés que algunos creadores cinematográficos comienzan a mostrar por localizaciones naturales como alternativa a los rodajes en los Estudios de Hollywood. No es hasta final de la década de los 60 cuando se registran las primeras iniciativas de colaboración entre la industria del cine y el territorio (Cucco y Richeri, 2013). Y es en este periodo cuando surgen estas agencias, que nacen, como apunta Eugeni Osácar, de la necesidad de articular "...una herramienta especializada para alcanzar el objetivo básico de captar rodajes y dinamizar el territorio” (Osácar, 2009, p. 19).

Este fenómeno empieza a extenderse por Europa a lo largo de la década de los 80 (Ghedini, 2007, p.30) y lo hace posteriormente, más de una década más tarde, por el resto del mundo. En la actualidad las FCs, presentes en todos los continentes, suelen estar organizadas mediante redes. Existe una red mundial, denominada Association Film Commissioner International, que agrupa a más de 300 Film Commissions, y que es el referente de esta actividad audiovisual a nivel global. Las demás se han establecido por regiones internacionales. Destacan entre ellas, European Film Commission Network, 
African Film Commissions Network, Asian Film Commissions Network y Latin American Film Commission Network-.

En Europa, como ya se ha apuntado, el desarrollo de las Film Commissions se ha producido en las tres últimas décadas. Desde el año 2007 las oficinas europeas de promoción de rodajes se encuentran organizadas en la European Network Film Commission, que acoge a cerca de 100 oficinas de 30 países. España es uno de los territorios integrado en la red. Lo hace a través de 17 agencias de diferentes ámbitos geográficos y administrativos (local, comarcal, provincial, regional, e incluso nacional) ${ }^{1}$. Una de las entidades que participa activamente en la red europea es la Spain Film Commission. Se trata de una asociación sin ánimo de lucro que, a su vez, reúne a 35 oficinas de distintas coberturas ${ }^{2}$ y que asume la representatividad del sector. Esta red nacional se constituyó en marzo de 2001 y, como señalan sus fundadores, "pese a que todos los miembros de Spain Film Commission han de pertenecer al sector público, SFC no está integrado en la estructura de las instituciones públicas representativas del Audiovisual" (Rosado y Querol, 2006, p. 84). De modo que es una asociación privada que cumple entre otras misiones con la:

"Interlocución con la administración central, territorial y municipal para potenciar la industria de rodajes; Colaboración con la administración para la creación de un sistema de incentivos fiscales eficiente; Apertura de nuevos mercados internacionales; e Impulso del turismo cinematográfico" (Spain Film Comission, 2017, s.p.).

En España, las primeras oficinas de rodajes se crearon en la década de los 90 del siglo XX. Canarias fue la región pionera en articular este tipo de instrumento para captar y explotar rodajes en el territorio. Le siguieron la ciudad de Barcelona y la Región de Andalucía (Rosado y Querol, 2006). En la actualidad existe al menos una Film Commission o Film Office (FO) con mayor o menor actividad, en cada una de las 17 comunidades autónomas.

La Film Commission es un actor de la cadena de valor de la industria audiovisual cuyo papel está marcado por su vocación de servicio a este sector. La entidad, que se desarrolla en el seno de una institución pública, tiene como principal misión apoyar el trabajo de una productora audiovisual externa o local desde el mismo momento en el que la compañía

\footnotetext{
${ }^{1}$ Andalucía Film Commission; Barcelona Film Commission; Bilbao Bizkaia Film Commission; Canary Islands Film; Cantabria Film Commission; Catalunya Film Commission; Film Madrid; Illes Balears Film Commission; Malaga Film Office; Mallorca Film Commission; Navarra Film Commission; Salamanca Film Commission; San Sebastian - Guipuzkoa Commission; Santiago de Compostela Film Commission; Spain Film Commission; Valencia Film Office; Zinealdea Film Bureau.

2 Andalucía Film Commission; Aragón Film Commission; Asturias Paraíso Natural Film Commission; Ávila Film Office; Benidorm Film Office; Bilbao Bizkaia Film Commission; Canary Islands Film; Cantabria Film Commission; Carmona Film Office; Castilla La Mancha Film Commission; Castilla y León Film Commission; Catalunya Film Commission; Ciudad de Madrid Film Office / City of Madrid Film Office; Extremadura Film Commission; Film Madrid; Illes Balears Film Commission; La Rioja Film Commission; Lleida Film Commission; Málaga Film Office; Mallorca Film Commission; Monegros Film Commission; Murcia Film Office; Navarra Film Commission; Salamanca Film Comission; Santiago de Compostela Film Commission; Segovia Film Office; Soria Film Commission; Terrasa Film Office - Parc Audiovisual de Catalaunya; Valencia Film Office; Valladolid Film Office; Victoria-Gateiz Film Office.
} 
manifiesta cierto interés por alguna de las localizaciones situadas en el territorio bajo su competencia.

A partir de ese primer acercamiento por parte del equipo de producción se desencadena toda una serie de acciones de la FC orientadas a lograr que este adopte la decisión de efectuar el rodaje en el territorio (Ramahí, 2011). Para ello la oficina, que se pone a disposición de la productora desde su llegada al territorio, le ofrece localizaciones acordes a las necesidades del guion o bien orienta su búsqueda; se brinda para gestionar los permisos de rodaje o ayudar a su tramitación; y se presta para acompañarla en cualquiera de sus tareas en la zona. Otra de las acciones fundamentales de la Film Commission, encaminada a retener ese rodaje, es informar a la compañía externa sobre el volumen, competencia y profesionalidad de los recursos técnicos a los que la productora podría acudir para completar el equipo de rodaje que tendría que desplazar a ese lugar.

\section{LOS RECURSOS TÉCNICOS Y PROFESIONALES AUDIOVISUALES COMO ATRACTIVO DEL TERRITORIO}

En la actividad de producción audiovisual la eficiencia es el principio que debe presidir toda la organización de un rodaje (Jacoste, 1996). El ahorro de costes, sin perder de vista la calidad, es una exigencia en la planificación y ejecución de cualquier proyecto audiovisual para garantizar su viabilidad, especialmente en el ámbito cinematográfico (Pardo, 2014). En este marco es en el que la relación de la Film Commission con la industria audiovisual local se estrecha hasta convertirse en esencial para ambas partes.

El atractivo de un territorio como espacio de rodaje no solo viene dado por el valor paisajístico y monumental de la zona, ni por la climatología, tampoco por la importancia del incentivo fiscal, sino también por otra razón. Cuando la localización se encuentra lejos de los polos de producción en los que se concentra la oferta de profesionales y empresas audiovisuales -como podrían ser Madrid, Barcelona entre otros-, el interés también reside en estos casos en la posibilidad de contratar personal cualificado y servicios audiovisuales de calidad en el propio territorio. Se evitaría así el desplazamiento de profesionales desde la ciudad o país desde donde opera la compañía, con el consecuente ahorro en dietas y alojamiento. Este es un aspecto al que los investigadores Marco Cucco y Giuseppe Richeri otorgan una gran importancia en la medida en la que se convierte en un criterio de elección de un espacio. En este sentido, estos autores consideran que "the possibility of using locals is therefore a real attraction for the production company as it allows a significant reduction in costs" (Cucco y Richeri, 2011, p. 8). De modo que, la existencia de profesionales y empresas audiovisuales en el territorio puede ser el requisito para que un municipio se transforme en una localización.

Esta circunstancia ha propiciado una relación simbiótica inigualable entre la Film Commission y el sector audiovisual local por dos motivos. De un lado, esta situación se explica por el hecho de que para que la agencia esté en disposición de persuadir a una productora de la pertinencia de elegir su localidad o región debería poder ofrecer, al margen de unas buenas localizaciones, también un mercado de técnicos y compañías de servicios capaz de asumir con garantías las necesidades de la productora. 
Por otro lado, esa vinculación responde a otro hecho. La Film Commission es un dispositivo planteado ex profeso para captar rodajes y, como consecuencia, para dinamizar la actividad audiovisual en el territorio. La presencia de producción externa, o incluso interna, supone, sin ninguna duda, la generación de empleo más o menos especializado, así como la contratación de empresas audiovisuales y auxiliares autóctonas. De modo que, la industria audiovisual local se ve de esta manera favorecida por las diligencias de la oficina fílmica.

En este contexto, por lo tanto, se produce esa relación de dependencia y beneficio mutuo entre la Film Commission y la industria audiovisual local en tanto que, de un lado, la oficina fílmica necesita de un sector audiovisual sólido como reclamo para lograr la elección del territorio como espacio de rodaje, y de otro, los profesionales y empresas locales encuentran en la Film Commission un instrumento de promoción de su sector ante las compañías ajenas al territorio, generador de nuevas oportunidades de participación en rodajes.

\section{LA DIFUSIÓN DE LA INFORMACIÓN SOBRE LOS ACTIVOS PROFESIONALES Y SERVICIOS AUDIOVISUALES DEL TERRITORIO}

Una de las funciones básicas de una comisión fílmica, según el profesor Roberto Bellas (2003), es, además de ofrecer información, posibilitar el contacto entre productores con empresas audiovisuales y con técnicos especializados en todos los ámbitos: desde electricistas, cámaras, decoradores, sonido, equipamiento, pasando por alquiler de vehículos, hasta asesoría financiera o laboral. De modo que, generalmente la Film Commission intenta abarcar todos los oficios y servicios susceptibles de ser contratados en el territorio por parte de una productora externa.

El principal mecanismo empleado por parte de las Film Commissions en la promoción del sector audiovisual local como valor añadido al resto de bondades de ese espacio es la difusión de la información de profesionales y empresas del territorio a través de su página web. Aunque algunas oficinas aplican también otros métodos para proyectar este activo, la presentación de los datos del personal técnico y empresas a través de la página web es el más extendido. Esta es la razón por la que el estudio se centra en el comportamiento de las Film Commissions respecto al uso de esta herramienta.

Para efectuar el análisis de las acciones de las oficinas se toma como referencia la relación de las 35 entidades registradas en la Spain Film Commission. Entre ellas hay trece agencias regionales -no disponen de este tipo de oficinas a nivel autonómico ni el País Vasco, ni Galicia o Valencia, que sí cuentan con Film Commissions provinciales o municipales, ni la Región de Murcia donde solo hay una oficina local-. Hay dos oficinas municipales que han ampliado su cobertura a la provincia. Se trata de la de Bilbao y de Vitoria-Gastéiz. Y, el resto de agencias, o bien son insulares -La Palma, Tenerife, Gomera, Lanzarote y Mallorca-; o bien comarcales - Monegros-, y locales - todas las demás. 
La información sobre profesionales y empresas suele presentarse en la página web de las Film Commission bajo diferentes denominaciones. Las etiquetas más frecuentes identificadas que dan acceso a los datos son: "Guía de producción"; "Guía audiovisual"; "Guía de profesionales”; y "Directorio profesional y de empresas".

En lo que respecta a qué oficinas ofrecen esos datos se observa que, en la esfera regional, todas las oficinas exponen la referencia de los recursos técnicos de su territorio, con la excepción de Extremadura, que no lo hace, pero pide a los interesados que se pongan en contacto con la oficina. Curiosamente en Cantabria sólo se aportan los datos de profesionales, mientras que, en la Comunidad de Madrid, solo se proporcionan los de empresas. En el resto de casos, las Film Commissions regionales ofrecen información tanto de profesionales como de empresas -Andalucía, Aragón, Asturias, C. Mancha, C. León, Cataluña, La Rioja y Navarra-.

A nivel provincial e insular, mientras la FC de Vitoria-Gastéiz facilita información tanto de personal como de compañías de servicios, las de Bilbao, La Palma y Tenerife sólo lo hacen sobre estas últimas. En Mallorca se ofrece un directorio de empresas, pero en lo referente a profesionales se remite al interesado a consultar la página web de la empresa de trabajo temporal, Adecco Audiovisual.

En cuanto a las oficinas locales, hay entidades que han hecho el esfuerzo de abrir un espacio de información de recursos locales tanto de técnicos como de compañías. Es el caso de las Film Offices de Málaga, Madrid, Valladolid, Benidorm, Santiago de Compostela y Valencia. Esta última oficina presenta una singularidad. En su sitio web no se aportan datos de profesionales de forma individual, sino que se facilitan los contactos de diez colectivos regionales de técnicos de diferentes especialidades a través de los que se accede a los datos de sus asociados.

Por otra parte, también hay que señalar que existen algunas oficinas locales e insulares que no prestan este servicio de información. Una de las razones es que todas ellas están vinculadas a oficinas de ámbito regional que sí brindan esos datos. Se trata de las Film Offices de Terrassa, Gomera y Lanzarote.

Con alguna excepción curiosa, como es el caso de Salamanca FO que sólo aporta información de empresas de logística, las oficinas suelen ofrecer información de profesionales y compañías de servicios audiovisuales y auxiliares -Cantabria FC, Valladolid FO, La Palma FC, Benidorm FO- o bien, como en el resto de casos, los contactos remiten a todo tipo de recursos susceptibles de ser empleados: personal y empresas tanto audiovisuales como auxiliares o de logística. Es decir, la oficina intenta poner a disposición de los interesados toda la información que pueda ser de utilidad para el director o jefe de producción encargado de la planificación y la contratación de personal y servicios en el territorio. Al facilitar esos contactos y, por lo tanto, quedar expuestos y visibles a cualquier compañía, se abre la posibilidad de que las empresas locales reciban ofertas de trabajo y encargo de servicios.

La forma más común de presentación de los datos suele ser mediante un motor de búsqueda por categorías, organizadas por especialidades o tipo de actividad. En 
determinadas oficinas se puede introducir, además, un filtro por provincias, como es el caso de Andalucía Film Commission, o por localidades como sucede en la Cataluña FC, Castilla La Mancha FC o La Rioja FC. Al permitir la búsqueda por municipios se potencia aún más la puesta en valor de los recursos a escala local, reforzando así la proyección del sector audiovisual de proximidad.

Además del buscador, hay otra opción para obtener información, basada en el acceso a los datos a través de un listado de contactos. Segovia FO, Valladolid FO, La Palma FC y Bilbao FO lo hacen así. En Cantabria se emplean las dos fórmulas, buscador y relación de profesionales y empresas. Y finalmente, el folleto en formato digital también es un formato al que recurre alguna oficina. Valencia FO y Tenerife son dos de ellas.

La presencia del personal técnico y artístico, así como de las empresas de servicios, es voluntaria. Si bien en algunas FCs es la propia entidad la que recaba la información y la expone - así se produce en Segovia, Valladolid, Salamanca, Valencia, Bilbao, La Rioja y Santiago de Compostela-, en todas las demás Film Commissions o Film Offices se brinda al interesado la posibilidad de dar sus datos y presentarse a través de una aplicación o formulario que es volcado a la base de datos y al buscador o listado con los que la FC contribuye a dar a conocer los activos audiovisuales, auxiliares y logísticos de su ámbito de cobertura (ver Tabla 1).

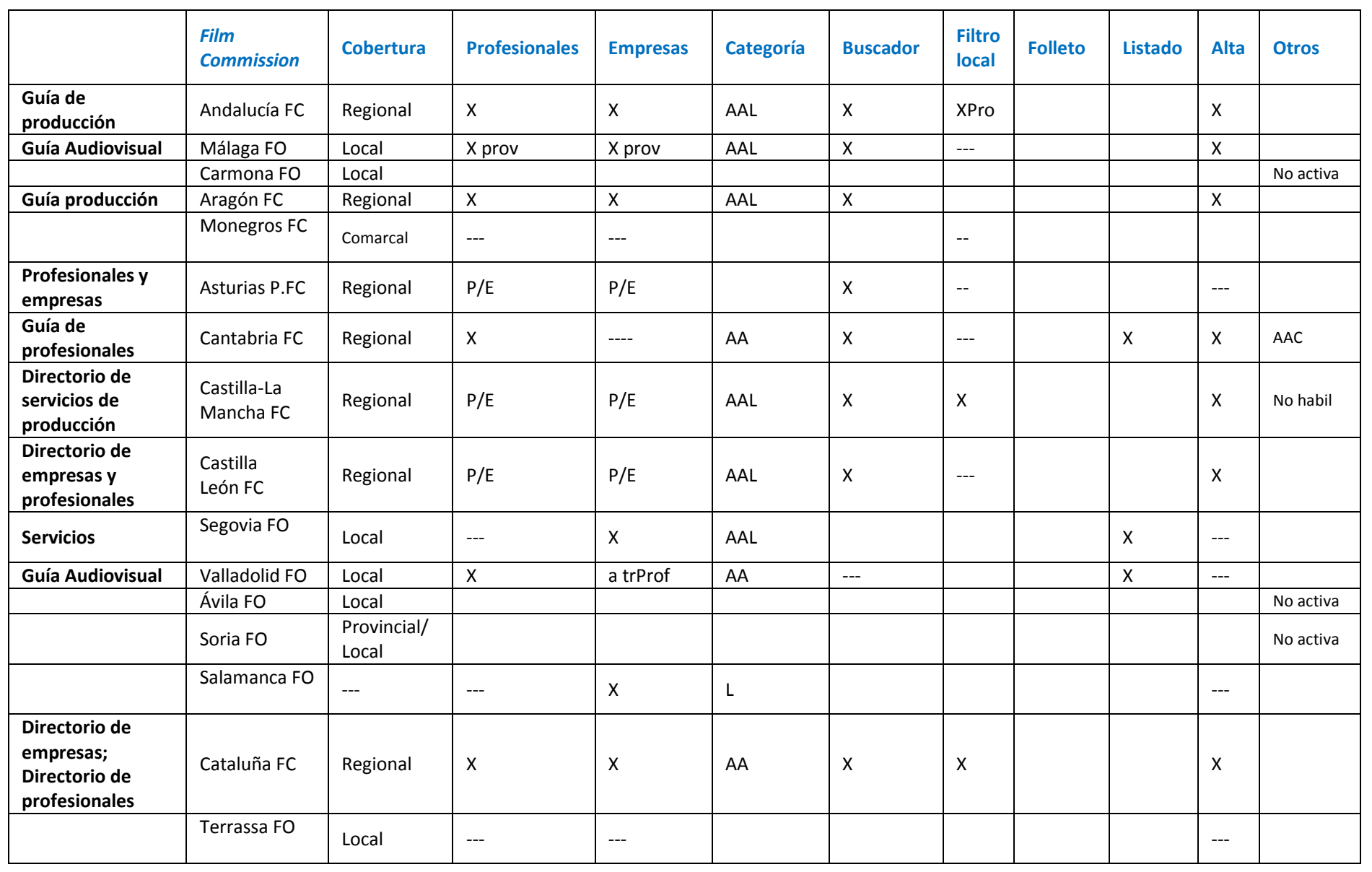




\begin{tabular}{|c|c|c|c|c|c|c|c|c|c|c|c|}
\hline & Lérida FO & Local & & & & & & & & & No activa \\
\hline $\begin{array}{l}\text { Directorio de } \\
\text { profesionales }\end{array}$ & Benidorm FO & Local & $x$ & algu & AA & $x$ & & & & $x$ & \\
\hline \multirow[t]{2}{*}{$\begin{array}{l}\text { Guía de } \\
\text { profesionales }\end{array}$} & Valencia FO & Local & $x$ & $x$ & --- & & & $x$ & & --- & Asoc A \\
\hline & $\begin{array}{l}\text { Extremadura } \\
\text { FC }\end{array}$ & Regional & -- & --- & --- & & & & & & Conta \\
\hline Empresas & Santiago CFO & Local & Adecco & $\mathrm{X}$ prov & AAL & $x$ & --- & & & --- & \\
\hline $\begin{array}{l}\text { Directorios } \\
\text { profesionales y } \\
\text { empresas }\end{array}$ & $\begin{array}{l}\text { Islas Baleares } \\
\text { FC }\end{array}$ & Regional & $P / E$ & $P / E$ & AAL & & Islas & & & $x$ & \\
\hline \multirow[t]{2}{*}{$\begin{array}{l}\text { Directorios } \\
\text { profesionales y } \\
\text { empresas }\end{array}$} & Mallorca FC & Isla & $P / E$ & $P / E$ & AAL & & $\begin{array}{l}\text { Mall } \\
\text { orca }\end{array}$ & & & $x$ & \\
\hline & $\begin{array}{l}\text { Gran Canaria } \\
\text { FC }\end{array}$ & Isla & & & & & & & & & No activa \\
\hline $\begin{array}{l}\text { Directorio de } \\
\text { empresas }\end{array}$ & La Palma FC & Isla & --- & $x$ & A & -- & & & $x$ & $\begin{array}{l}X \\
\text { Prof } \\
\end{array}$ & \\
\hline \multirow[t]{3}{*}{$\begin{array}{l}\text { Directorio } \\
\text { profesional }\end{array}$} & Tenerife FC & Isla & & $x$ & AAL & $x$ & & $x$ & & --- & \\
\hline & Gomera FC & Isla & --- & --- & & & & & & & Activa \\
\hline & Lanzarote FC & Isla & --- & --- & & & & & & & Activa \\
\hline Guía producción & La Rioja FC & Regional & $\mathrm{P} / \mathrm{E}$ & $\mathrm{P} / \mathrm{E}$ & AAL & & $x$ & & & $-\ldots$ & \\
\hline Guía Audiovisual & Film Madrid & Regional & $-\ldots$ & $x$ & AAL & $x$ & & & & $x$ & \\
\hline $\begin{array}{l}\text { Directorios } \\
\text { profesionales y } \\
\text { empresas }\end{array}$ & Madrid FO & Local & $x$ & $x$ & AA & $x$ & & & & $\mathrm{x}$ & \\
\hline Guía producción & Navarra FC & Regional & $x$ & $x$ & AAL & $x$ & & & & $x$ & \\
\hline Guía producción & Bilbao FC & $\begin{array}{l}\text { Provincial/ } \\
\text { Local }\end{array}$ & --- & $x$ & $\mathrm{AA}$ & & & & $x$ & --- & \\
\hline \multirow[t]{2}{*}{ Guía producción } & Vitoria FC & $\begin{array}{l}\text { Provincial/ } \\
\text { Local }\end{array}$ & $x$ & $x$ & AA & & & & $x$ & $x$ & \\
\hline & Murcia FO & Local & & & & & & & & & No activa \\
\hline
\end{tabular}

Tabla 1. El Servicio de información sobre recursos audiovisuales y otros en las oficinas adheridas a la Spain Film Commissions. Fuente: Elaboración propia a partir de la información obtenida de las webs de las 35 oficinas (2019)

\section{ESTUDIO DE CASO EN ESPAÑA: LA ANDALUCÍA FILM COMMISSION (AFC)}

La Andalucía Film Commission (AFC) es una red de oficinas de promoción de rodajes constituida en 1998 “...bajo los auspicios de la Empresa pública de la Radiotelevisión de Andalucía, a través de su fundación especializada en el fomento del audiovisual andaluz" (Rosado y Querol, 2006, p. 91). En 2019 integra en torno a 70 oficinas municipales, comarcales y provinciales. Esta red, a su vez, forma parte de otras entidades de mayor cobertura. Es miembro de la Spain Film Commission, así como de la European Film Commission Network, y de la Association of Film Commissioners International.

Los miembros de la Andalucía Film Commission ofrecen, por medio de la red, servicios en torno a dos principales ejes. Uno de ellos es el suministro de información. Además de mantener al día a los productores locales y externos en materia de ayudas, convocatorias y subvenciones activas, otra de las misiones de estas entidades en Andalucía es informar sobre espacios susceptibles de ser utilizados para rodajes. Para ello la asociación cuenta con un completo catálogo de localizaciones (públicas y privadas), que se brinda y canaliza a través de la página web de la Andalucía FC o, además, a través de la propia oficina 
integrada en la red, como sucede con la Málaga FO. Por otra parte, las agencias facilitan datos de contacto para la contratación por parte de las productoras acogidas en el territorio, tanto de empresas de servicios especializados o de logística (alojamiento, restauración, alquiler de vehículos) como de profesionales del audiovisual establecidos en Andalucía.

El segundo eje que marca la actuación de la Andalucía Film Commission es su misión como enlace entre las productoras externas y las instituciones públicas del territorio que las recibe. La relación de la AFC con los diferentes departamentos de la administración otorga un gran valor a la oficina en el desarrollo de determinadas gestiones. En este sentido, las entidades que integran la Andalucía Film Commission suelen asesorar a las productoras en materia de solicitud de permisos. Incluso en algunos casos, se ocupan ellas mismas de la gestión y seguimiento de una autorización en el seno de su municipio o diputación, y se convierten en las interlocutoras entre la administración y la empresa audiovisual acogida en el territorio.

A cambio de la prestación de estos servicios gratuitos, las oficinas andaluzas piden la inclusión de su logotipo y una mención en los títulos de crédito de la producción resultante, así como, si procede, la cesión de materiales de rodaje y datos, que el municipio pudiera explotar para la promoción de su territorio, o bien como potencial espacio de rodajes para otras productoras, o bien como atractivo en términos de turismo cinematográfico.

En lo que se refiere al objeto de estudio de esta investigación, la relación de la Film Commission con la industria audiovisual local, la AFC proporciona visibilidad a todas las empresas y profesionales del ámbito audiovisual que lo deseen a través de su página web, con el fin de proporcionar los recursos técnicos necesarios para atraer rodajes a Andalucía y facilitar su ejecución.

Los interesados pueden darse de alta de manera online y de forma voluntaria en la propia plataforma. La experiencia de usuario es sencilla, solo es necesario acceder a "Guía de producción". Posteriormente se abre una segunda ventana con dos pestañas, una de ellas indica "Alta", y dentro de ella se discrimina entre "Alta de profesionales" y "Alta de empresas".

Del mismo modo, dentro de la etiqueta "Guía de producción" se llega al "buscador" que da acceso a las 117 categorías que ofrece la web de la Andalucía Film Commission. Estos apartados se organizan en torno a dos grandes bloques: por un lado, "1. Profesionales", que cuenta con 20 especialidades, que a su vez se subdividen en diferentes tipos de profesionales; y, por otro lado, "2. Empresas", con 8 especialidades, que también se dividen en distintas tipologías.

El fácil acceso a la información en la página web de AFC, sin duda, proporciona visibilidad a los activos audiovisuales autóctonos y contribuye a su posible contratación por parte de producciones extranjeras. Por tanto, si se establece una relación entre este escaparate y el número de contrataciones de profesionales del sector audiovisual vinculados a rodajes en el territorio andaluz en un periodo de cinco años, se constata que el nivel de empleabilidad de profesionales andaluces ha ido creciendo a lo largo de los años. Por ejemplo, desde el 
año 2013 hasta el 2017 se ha producido un alto nivel de contratación, entre puestos directos, indirectos e inducidos. Se trata de trabajo cualificado de técnicos del audiovisual y servicios e industrias conexas. La suma asciende a 82.815 trabajadores empleados. El número de contrataciones ha ido aumentado proporcionalmente año tras año. En $2013 \mathrm{y}$ 2014 se emplearon a más de 14.300 trabajadores; en el año 2015 se contrataron a 16.699 profesionales relacionados con la producción audiovisual; el año 2016 sufrió un leve retroceso y alcanzó los 15.541 trabajadores contratados; sin embargo, en el año 2017, las cifras se incrementaron notablemente y ascendieron a los 21.877 profesionales empleados (AFC, Informes de Actividad 2013, 2014, 2015, 2016 y 2017).

\subsection{Profesionales de producción audiovisual en Andalucía Film Commission (AFC)}

Los profesionales vinculados a la producción audiovisual están etiquetados con "1. Profesionales" y dentro de esta categoría se encuentran 20 especialidades: 1.1. Animador; 1.2. Arte; 1.3. Coreógrafo; 1.4. Diseñador gráfico; 1.5. Dirección/realizador; 1.6. Documentalista; 1.7. Doblador; 1.8. Efectos digitales; 1.9. Efectos especiales; 1.10. Especialista; 1.11. Fotografía; 1.12. Guionista; 1.13. Maquillaje/peluquería; 1.14. Montaje; 1.15. Música; 1.16. Producción; 1.17. Sonido; 1.18. Storyboard; 1.19. Vestuario; 1.20. Foto fija. Cada una de estas especialidades se subdivide en diferentes subcategorías. Para esta investigación se han seleccionado las 30 especialidades que tienen datos desglosados por provincias. La posibilidad de poder localizar a cada uno de estos profesionales por provincias agiliza las búsquedas y facilita las tareas de los jefes de producción.

En concreto, AFC ofrece información de un total de 801 profesionales distribuidos por las ocho provincias andaluzas. Tal y como podemos ver en la Tabla 2, la de Sevilla ostenta el mayor número de técnicos audiovisuales especializados. En concreto, 288 profesionales están registrados en la página web en esta ciudad. Sevilla es la capital de la Comunidad Autónoma de Andalucía y alberga la sede de la televisión autonómica, por lo tanto, concentra la mayor actividad audiovisual de la región.

A través de la página web de AFC, se constata también que Málaga es la segunda provincia con más profesionales registrados, ya que ofrece información de 155 especialistas audiovisuales. Le sigue Granada con 113 profesionales y Almería con 109. Un tanto alejadas se encuentran las otras cuatro provincias: Cádiz con 85; Córdoba con 27; Jaén con 15; y Huelva con 9.

\begin{tabular}{|l|c|c|c|c|c|c|c|c|c|}
\hline \multirow{2}{*}{ PROFESIONALES } & Almería & Cádiz & Córdoba & Granada & Huelva & Jaén & Málaga & Sevilla & TOTAL \\
\cline { 2 - 9 } & 109 & 85 & 27 & 113 & 9 & 15 & 155 & 288 & $\mathbf{8 0 1}$ \\
\hline
\end{tabular}

Tabla 2. Profesionales por provincias disponibles en www.andaluciafilm.com. Fuente: Elaboración propia a partir de la información obtenida de la página web de Andalucía Film Commission (2019)

Si se tiene en cuenta el funcionamiento de una producción audiovisual, se puede comprobar que Andalucía Film Commission brinda la posibilidad de contratar todo tipo de profesionales en cualquier fase del proceso para sacar adelante un producto audiovisual tanto cinematográfico, como televisivo, o publicitario, etc. (Ver Tabla 3) 
Para la creación de un guion se puede conseguir que hasta 21 documentalistas orienten el trabajo y, que hasta 54 guionistas puedan darle forma y transformen la idea en un guion literario.

Para la fase de producción, todas las categorías de profesionales vinculados con esta etapa están representados en la página web y pueden ser contratados: arte (15); directores artísticos (35); ayudantes de dirección artística (37), atrezzistas (32); ayudantes de atrezzo (21); coreógrafos (3); diseñadores gráficos (30).

En lo que se refiere a la fase de dirección, se puede montar un equipo completo de profesionales que atienda todos los puestos: 74 directores/realizadores; 52 ayudantes de dirección/realizador; 53 ayudantes de dirección/realización; 15 scripts; 8 regidores; 7 ayudantes de regiduría; 42 expertos en fotografía; 18 especialistas en efectos especiales; 74 operadores de cámara; 33 ayudantes de cámara; 39 auxiliares de cámara; 18 vídeo asistentes; 13 operadores de steadicam; 9 jefes de eléctricos; 21 eléctricos; 5 maquinistas.

Para la fase de postproducción y cierre de un proyecto, se cuenta con la disponibilidad de 29 editores; 13 animadores 3D; 12 especialistas en efectos digitales; y 12 dobladores/locutores.

\begin{tabular}{|c|c|c|c|c|c|c|c|c|c|}
\hline PROFESIONALES & Almería & Cádiz & Córdoba & Granada & Huelva & Jaén & Málaga & Sevilla & \\
\hline Animador 3D & 2 & 2 & 0 & 0 & 0 & 0 & 3 & 6 & 13 \\
\hline Arte & 3 & 2 & 1 & 1 & 0 & 0 & 2 & 6 & 15 \\
\hline Director artístico & 3 & 3 & 0 & 4 & 1 & 0 & 10 & 14 & 35 \\
\hline $\begin{array}{l}\text { Ayudante de dirección } \\
\text { artística }\end{array}$ & 3 & 6 & 1 & 8 & 1 & 0 & 4 & 14 & 37 \\
\hline Atrezzista & 4 & 2 & 1 & 6 & 0 & 0 & 4 & 15 & 32 \\
\hline Ayudante de atrezzo & 5 & 4 & 0 & 3 & 1 & 1 & 2 & 5 & 21 \\
\hline Regidor & 1 & 1 & 0 & 4 & 0 & 0 & 2 & 1 & 8 \\
\hline Ayudante de regiduría & 0 & 0 & 0 & 2 & 1 & 0 & 0 & 4 & 7 \\
\hline Coreógrafo & 0 & 0 & 0 & 1 & 0 & 0 & 1 & 1 & 3 \\
\hline Diseñador gráfico & 4 & 7 & 2 & 4 & 0 & 1 & 5 & 7 & 30 \\
\hline Director/realizador & 8 & 9 & 5 & 9 & 1 & 2 & 11 & 29 & 74 \\
\hline $\begin{array}{l}\text { Ayudante de } \\
\text { Dirección/realización }\end{array}$ & 5 & 5 & 2 & 5 & 0 & 0 & 16 & 19 & 52 \\
\hline $\begin{array}{l}\text { Auxiliar de dirección/ } \\
\text { realización }\end{array}$ & 7 & 5 & 2 & 7 & 0 & 1 & 10 & 21 & 53 \\
\hline Script & 2 & 2 & 0 & 2 & 0 & 0 & 4 & 5 & 15 \\
\hline Documentalista & 2 & 1 & 0 & 5 & 1 & 1 & 1 & 10 & 21 \\
\hline Doblador/Locutor & 1 & 1 & 3 & 2 & 0 & 0 & 0 & 5 & 12 \\
\hline Efectos digitales & 2 & 2 & 1 & 1 & 0 & 0 & 3 & 3 & 12 \\
\hline Efectos especiales & 4 & 3 & 0 & 4 & 0 & 0 & 2 & 5 & 18 \\
\hline Especialista & 2 & 0 & 0 & 0 & 0 & 0 & 0 & 4 & 6 \\
\hline Fotografía & 3 & 7 & 2 & 7 & 0 & 1 & 12 & 10 & 42 \\
\hline Operador de cámara & 11 & 6 & 1 & 13 & 1 & 3 & 19 & 20 & 74 \\
\hline Ayudante de cámara & 7 & 1 & 0 & 8 & 0 & 0 & 8 & 9 & 33 \\
\hline Auxiliar de cámara & 8 & 3 & 0 & 5 & 0 & 1 & 8 & 14 & 39 \\
\hline Video Asistente & 6 & 0 & 0 & 2 & 0 & 0 & 6 & 4 & 18 \\
\hline
\end{tabular}




\begin{tabular}{|l|r|r|r|r|r|r|r|r|r|}
\hline Steadicam & 1 & 2 & 1 & 2 & 0 & 0 & 3 & 4 & $\mathbf{1 3}$ \\
\hline Jefe de eléctricos & 0 & 0 & 0 & 0 & 0 & 0 & 3 & 6 & $\mathbf{9}$ \\
\hline Eléctrico & 5 & 2 & 0 & 0 & 0 & 0 & 5 & 9 & $\mathbf{2 1}$ \\
\hline Maquinista & 1 & 0 & 0 & 0 & 0 & 0 & 1 & 3 & $\mathbf{5}$ \\
\hline Guionista & 5 & 5 & 3 & 3 & 2 & 2 & 8 & 26 & $\mathbf{5 4}$ \\
\hline Editor & 4 & 4 & 2 & 5 & 0 & 2 & 2 & 10 & $\mathbf{2 9}$ \\
\hline & $\mathbf{1 0 9}$ & $\mathbf{8 5}$ & $\mathbf{2 7}$ & $\mathbf{1 1 3}$ & $\mathbf{9}$ & $\mathbf{1 5}$ & $\mathbf{1 5 5}$ & $\mathbf{2 8 8}$ & $\mathbf{8 0 1}$ \\
\hline
\end{tabular}

Tabla 3. Profesionales desglosados por especialidades y distribuidos por provincias disponibles en www.andaluciafilm.com Fuente: Elaboración propia a partir de la información obtenida de la página web de Andalucía Film Commission (2019)

\subsection{Empresas de producción audiovisual en Andalucía Film Commission (AFC)}

Dentro del apartado "2. Empresas" se pueden encontrar 8 especialidades vinculadas a este sector: 2.1. Alquiler de equipos y material; 2.2. Distribución; 2.3. Escuelas de Artes Escénicas; 2.4. Exhibición; 2.5. Facultades y Escuelas Audiovisuales; 2.6. Productoras; 2.7. Servicios a la producción; 2.8. Servicios logísticos. En este apartado de la investigación se presta atención exclusivamente al apartado 2.6. destinado a las Productoras.

Tal y como se ha expuesto en líneas anteriores, una empresa productora es responsable del desarrollo y grabación o filmación de un proyecto audiovisual. AFC ofrece un amplio directorio de empresas de esta especialidad a través de su página web. Se trata de compañías asentadas en el territorio que, además de generar sus propios productos, brindan sus servicios a producciones extranjeras. La fórmula contractual es muy variada y depende de las necesidades del solicitante, que puede demandar desde el alquiler de equipamiento técnico, hasta el contrato de determinados profesionales.

Andalucía Film Commission ha dividido a las empresas productoras en tres tipologías: 1. Cine; 2. Publicidad; 3. Televisión; y ha dejado una cuarta categoría etiquetada como "otros". En su página web, se dispone de información de un total de 123 empresas productoras audiovisuales; 36 de ellas se dedican a producción cinematográfica; 37 son productoras de publicidad; 39 son empresas de televisión; y 11 están etiquetadas como "otros".

La información de cada categoría se ofrece también diversificada por provincias: Almería, Cádiz, Córdoba, Granada, Huelva, Jaén, Málaga y Sevilla. Además de los datos correspondientes a nombre de la productora, dirección postal, teléfono y correo electrónico, el usuario dispone de información añadida de la empresa, ya que se proporciona un listado de sus principales trabajos audiovisuales.

Si se observa en la Tabla 4 la distribución por provincias, se constata que Sevilla alberga a la mayoría de las empresas productoras de cine. Casi la mitad de las productoras de cine (15) tiene su sede social en la capital de la Comunidad Autónoma. Le sigue Málaga con 6, Almería con 5 y Granada con 4. No obstante, llama la atención que en todas las provincias andaluzas haya, al menos, una productora de cine. Así, en Cádiz y en Córdoba hay 2 productoras de cine; y 1 en Huelva y 1 en Jaén. 
Con respecto a las productoras de publicidad, el mayor número de empresas se concentra también en Sevilla con 15 productoras; le sigue Málaga con 10. De nuevo, casi todas las provincias cuentan con productoras de publicidad, con la excepción de la provincia de Córdoba. Por lo tanto, tal y como se refleja en la tabla, Cádiz cuenta con 4 productoras de publicidad; Almería con 3 empresas; Granada y Huelva con 2 compañías; y Jaén con 1 productora de publicidad.

El sector de la producción de televisión proporciona el mayor número de empresas. La Andalucía Film Commission ofrece datos de 39. De nuevo, en esta ocasión, el sector se concentra en Sevilla. La capital acoge a 18 productoras; y le sigue Málaga con 7 compañías de televisión. Todas las provincias tienen al menos una productora: Almería (4); Cádiz y Granada (3); Córdoba (2); Huelva y Jaén (1).

La página web de Andalucía Film Commission facilita un apartado de "otros". Probablemente, se trate de productoras audiovisuales que ofrezcan servicios múltiples y que han decidido no adherirse sólo a una "etiqueta". En este apartado se ubican 11 empresas; 4 en Sevilla y 4 en Almería; 1 en Málaga, 1 en Cádiz y 1 en Córdoba; y ninguna en Granada, Huelva y Jaén.

\begin{tabular}{|l|c|c|c|c|c|c|c|c|c|}
\hline PRODUCTORAS & Almería & Cádiz & Córdoba & Granada & Huelva & Jaén & Málaga & Sevilla & TOTAL \\
\hline Cine & 5 & 2 & 2 & 4 & 1 & 1 & 6 & 15 & $\mathbf{3 6}$ \\
\hline Publicidad & 3 & 4 & 0 & 2 & 2 & 1 & 10 & 15 & $\mathbf{3 7}$ \\
\hline Televisión & 4 & 3 & 2 & 3 & 1 & 1 & 7 & 18 & $\mathbf{3 9}$ \\
\hline Otros & 4 & 1 & 1 & 0 & 0 & 0 & 1 & 4 & $\mathbf{1 1}$ \\
\hline & $\mathbf{1 6}$ & $\mathbf{1 0}$ & $\mathbf{5}$ & $\mathbf{9}$ & $\mathbf{4}$ & $\mathbf{3}$ & $\mathbf{2 4}$ & $\mathbf{5 2}$ & $\mathbf{1 2 3}$ \\
\hline
\end{tabular}

Tabla 4. Tipos de productoras por provincias disponibles en www.andaluciafilm.com. Fuente: Elaboración propia a partir de la información obtenida de la página web de Andalucía Film Commission (2019)

5.3 Empresas de servicios a la producción en Andalucía Film Commission (AFC)

Para la elaboración de este apartado, se ha tenido en cuenta la categoría “2.7. Servicios a la producción" registrada en la página web de AFC. La función de una empresa de servicios consiste en el acompañamiento, apoyo y aprovisionamiento a un sector específico de la industria. Por lo tanto, las empresas de servicios a la producción tienen una labor fundamental para el mercado audiovisual, ya que se constituyen como facilitadores de equipamiento técnico y de productos auxiliares necesarios para llevar a cabo un rodaje.

La página web de Andalucía Film Commission suministra al usuario el acceso a la información de 198 empresas de servicios a la producción audiovisual (ver Tabla 5).

Las agencias de localizaciones ocupan un papel muy destacado en el marco del sector servicios a la producción audiovisual. De hecho, en la página web de Andalucía Film Commission se pueden contabilizar hasta 39 empresas; le siguen las agencias de castings con 32 empresas; y las agencias de figuración con 25. Otro sector de actividad que 
predomina es el que pone a disposición de la producción audiovisual el alquiler de equipos y material audiovisual. Las empresas que se dedican a alquilar equipamiento técnico, y que están registradas en la página web de la Andalucía Film Commission son 30. Las empresas de peluquería y maquillaje también son numerosas. En la web se encuentran registradas hasta 22.

Por otro lado, también es posible acceder a la información de hasta 18 empresas de decorados y atrezzo; o de 15 compañías que proveen de vestuario. Las empresas de alquiler de vehículos equipados también juegan un papel destacado en una producción y 13 de ellas están disponibles en la página web de la AFC.

Si bien las empresas de catering son muy necesarias en cualquier producción audiovisual, sin embargo, en AFC solo se oferta información de 3 de ellas.

La provincia de Sevilla aglutina de nuevo el mayor número de empresas de servicios a la producción audiovisual. En total 72 empresas ubicadas en la capital están disponibles en la página web de Andalucía Film Commission. La provincia de Almería ocupa la segunda posición y en su territorio se pueden encontrar 39 empresas de servicios. La provincia de Málaga alberga 29 empresas; y le sigue la provincia de Cádiz con 23 empresas de servicios a la producción audiovisual.

\begin{tabular}{|c|c|c|c|c|c|c|c|c|c|}
\hline $\begin{array}{l}\text { SERVICIOS } \\
\text { PRODUCCIÓN }\end{array}$ & Almería & Cádiz & Córdoba & Granada & Huelva & Jaén & Málaga & Sevilla & TOTAL \\
\hline Figuración & 2 & 1 & 0 & 4 & 1 & 0 & 4 & 13 & 25 \\
\hline Localizaciones & 9 & 5 & 0 & 1 & 2 & 6 & 6 & 10 & 39 \\
\hline Castings & 6 & 2 & 1 & 4 & 1 & 0 & 5 & 13 & 32 \\
\hline $\begin{array}{l}\text { Decorados } \\
\text { y atrezzo }\end{array}$ & 3 & 1 & 0 & 2 & 0 & 2 & 4 & 6 & 18 \\
\hline Vestuario & 3 & 3 & 1 & 1 & 0 & 0 & 1 & 6 & 15 \\
\hline $\begin{array}{l}\text { Peluquería } \\
\text { y maquillaje }\end{array}$ & 5 & 8 & 0 & 1 & 0 & 0 & 2 & 6 & 22 \\
\hline $\begin{array}{l}\text { Alquiler de } \\
\text { equipos y material } \\
\text { audiovisual }\end{array}$ & 7 & 3 & 2 & 3 & 3 & 0 & 0 & 12 & 30 \\
\hline $\begin{array}{l}\text { Alquiler de vehículos } \\
\text { equipados }\end{array}$ & 3 & 0 & 0 & 0 & 0 & 0 & 5 & 5 & 13 \\
\hline \multirow[t]{2}{*}{ Catering } & 1 & 0 & 0 & 0 & 0 & 0 & 2 & 1 & 4 \\
\hline & 39 & 23 & 4 & 16 & 7 & 8 & 29 & 72 & 198 \\
\hline
\end{tabular}

Tabla 5. Tipos de empresas de servicios a la producción por provincias en www.andaluciafilm.com. Fuente: Elaboración propia a partir de la información obtenida de la página web de Andalucía Film Commission (2019) 


\subsection{Empresas de servicios logísticos en Andalucía Film Commission (AFC)}

Este epígrafe se ha elaborado con la información obtenida de la etiqueta "2.8. Servicios logísticos" disponible en la página web de AFC. Las empresas complementarias y las compañías de servicios logísticos también tienen un papel muy destacado en el rodaje de un producto audiovisual, ya que proporcionan el soporte básico para poder planificar y gestionar una producción. Los gestores de Andalucía Film Commission son muy conscientes de la necesidad de ofrecer toda la información posible sobre este tipo de empresas a través de su web.

Las empresas de servicios logísticos y complementarios que están disponibles en la página web de Andalucía Film Commission están organizadas en nueve categorías: agencias de viajes; alquiler de vehículos; banco; comunicaciones marítimas; embajada; empresas de seguridad; mensajería; oficina de turismo; parques naturales. Y también se posibilita la búsqueda por provincias. No obstante, si bien todas estas categorías están etiquetadas, la página web no siempre facilita información sobre ellas.

De las nueve categorías anunciadas, y desglosadas por provincias, se puede encontrar información en la Tabla 6 de seis de ellas: agencias de viajes (5); alquiler de vehículos (8); comunicaciones marítimas (2); empresas de seguridad (1); oficinas de turismo (1); y parques naturales (1).

\begin{tabular}{|c|c|c|c|c|c|c|c|c|c|}
\hline $\begin{array}{l}\text { SERVICIOS } \\
\text { LOGÍSTICOS }\end{array}$ & Almería & Cádiz & Córdoba & Granada & Huelva & Jaén & Málaga & Sevilla & TOTAL \\
\hline $\begin{array}{l}\text { Agencias } \\
\text { de viajes }\end{array}$ & 1 & 0 & 0 & 0 & 0 & 1 & 1 & 2 & 5 \\
\hline $\begin{array}{l}\text { Alquiler } \\
\text { de vehículos }\end{array}$ & 2 & 0 & 0 & 0 & 0 & 0 & 3 & 3 & 8 \\
\hline Bancos & 0 & 0 & 0 & 0 & 0 & 0 & 0 & 0 & 0 \\
\hline $\begin{array}{l}\text { Comunicaciones } \\
\text { marítimas }\end{array}$ & 2 & 0 & 0 & 0 & 0 & 0 & 0 & 0 & 2 \\
\hline Embajadas & 0 & 0 & 0 & 0 & 0 & 0 & 0 & 0 & 0 \\
\hline $\begin{array}{l}\text { Empresas } \\
\text { de seguridad }\end{array}$ & 0 & 0 & 0 & 0 & 0 & 0 & 0 & 1 & 1 \\
\hline $\begin{array}{l}\text { Empresas } \\
\text { de mensajería }\end{array}$ & 0 & 0 & 0 & 0 & 0 & 0 & 0 & 0 & 0 \\
\hline $\begin{array}{l}\text { Oficinas } \\
\text { de turismo }\end{array}$ & 1 & 0 & 0 & 0 & 0 & 0 & 0 & 0 & 1 \\
\hline \multirow[t]{2}{*}{$\begin{array}{l}\text { Parques } \\
\text { naturales }\end{array}$} & 0 & 0 & 0 & 0 & 0 & 0 & 0 & 1 & 1 \\
\hline & 6 & 0 & 0 & 0 & 0 & 1 & 4 & 7 & 18 \\
\hline
\end{tabular}

Tabla 6. Tipos de empresas de servicios logísticos por provincias en www.andaluciafilm.com. Fuente: Elaboración propia a partir de la información obtenida de la página web de Andalucía Film Commission (2019)

\section{CONCLUSIONES}

La relación entre las Film Commissions y la industria audiovisual de su ámbito de cobertura está basada tanto en el apoyo como en el beneficio mutuo. La reciprocidad se produce por el hecho de que, por una parte, una oficina fílmica, en su tarea de atraer rodajes, debe estar en disposición de ofrecer un mercado de profesionales y empresas 
audiovisuales de garantía para cubrir las necesidades de las productoras acogidas en el territorio. Estos recursos representan, sin lugar a dudas, un valor añadido al resto de atractivos del lugar y puede ser determinante en la toma de decisiones de un equipo de producción. Pero, por otra parte, la actividad de la industria audiovisual local se ve asimismo favorecida por la actuación de la Film Commission, que no solo se ocupa de promocionar y dar visibilidad al activo audiovisual, auxiliar y logístico local, sino que al lograr retener rodajes en el territorio propicia la contratación de personal y servicios audiovisuales en su jurisdicción para beneficio del sector audiovisual local.

La difusión a través de la página web de la información de profesionales y empresas del territorio es la fórmula más utilizada por parte de las Film Commissions para promocionar el sector audiovisual local. Aunque algunas oficinas utilizan otros modos complementarios de promocionar este activo.

El acceso a los datos sobre profesionales y empresas es claro y directo en todas las Film Commissions estudiadas. Los usuarios pueden encontrar fácilmente etiquetas identificativas con diversas denominaciones: "Guía de producción"; "Guía audiovisual”; "Guía de profesionales"; y "Directorio profesional y de empresas". Los motores de búsqueda permiten acceder a la información a través de la selección de diferentes categorías debidamente organizadas por especialidades o tipo de actividad. Además, en determinadas Film Commissions se puede introducir un filtro por provincias, como ocurre en el caso de Andalucía Film Commission; o por localidades, como sucede en las oficinas de Castilla-La Mancha, de Cataluña o La Rioja.

Para dar a conocer los recursos audiovisuales, auxiliares y logísticos de un territorio, la mayoría de Film Commissions brinda a los interesados la posibilidad de proporcionar sus datos a través de una aplicación o un formulario, que posteriormente es volcado a la base de datos y al buscador del sistema. Se trata de una acción voluntaria. En otros casos, es la propia Film Commission la que recaba la información y la expone. En el caso de Andalucía Film Commission, en la página web se pueden encontrar dos tipos diferentes de formulario on line, uno para dar de alta a profesionales, y otro para dar de alta a empresas, y son los propios profesionales y las empresas los que lo solicitan.

A través de la etiqueta "Guía de producción", Andalucía Film Commission (AFC) proporciona un completo catálogo, organizado en 117 categorías, de recursos audiovisuales, por lo que posibilita el acceso a la información para su posible contratación de todas las especialidades profesionales necesarias para llevar a cabo una producción audiovisual completa, desde la creación del guion, pasando por la ejecución de la dirección, hasta la edición y postproducción del proyecto. En todas las categorías estudiadas relacionadas con "profesionales de producción", "empresas de producción", "empresas de servicios a la producción", y "empresas de servicios logísticos", la provincia de Sevilla concentra el mayor número de figuras profesionales vinculadas al sector audiovisual.

En definitiva, la web de una Film Commission, como plataforma de visibilidad de alcance internacional de los recursos técnicos y profesionales propios, es esencial para demostrar 
la capacidad de un territorio de acoger rodajes. En este sentido, esta investigación ha puesto en evidencia la facultad de la que disponen las Film Commissions en España para poner en valor estos recursos audiovisuales y para convertirse en agentes dinamizadores de la industria audiovisual de su ámbito de cobertura. Y, por lo tanto, se demuestra que la Film Commission y la industria audiovisual local mantienen una fuerte relación simbiótica que debe preservarse y consolidarse.

\section{REFERENCIAS}

AFCI. (2019). Association of Film Commissioners International. Recuperado de https://www. https://afci.org/

Andalucía Film Commission. (2013). Informe de actividad 2013. Recuperado de http://www.andaluciafilm.com

Andalucía Film Commission. (2014). Informe de actividad 2014. Recuperado de http://www.andaluciafilm.com

Andalucía Film Commission. (2015). Informe de actividad 2015. Recuperado de http://www.andaluciafilm.com

Andalucía Film Commission. (2016). Informe de actividad 2016. Recuperado de http://www.andaluciafilm.com

Andalucía Film Commission. (2017). Informe de actividad 2017. Recuperado de http://www.andaluciafilm.com

Bellas, R. (2003). Consideraciones sobre estructura, funciones y operativa de las comisiones fílmicas. En M. Martínez (dir.), Las comisiones fílmicas. Un dispositivo para la promoción audiovisual. Sevilla: Comunicación Social.

Cucco, M. y Richeri, G. (2011). Film commission as a driver for economic and cultural development. Conference Proceedings. Fourth Euro-Mediterranean Dialogue on Public Management. Rabat.

Cucco, M. y Richeri, G. (2013). Il mercato delle location cinematografiche. Venezia: Saggi Marsilio.

Figueira, A.P., Figueira, V. y Monteiro, S. (2015). Tourism and cinema: the importance of a film commission in promoting the destination Aletenjo. International Journal of Scientific Management Tourism, 3, pp. 29-37.

Ghedini, O.P. (2007). Le Film commission: esperienze e prospettive, le situazioni del federalismo. Rivista Studi giuridici e politici della Regione Emilia Romagna, 4, pp.29-37.

Jacoste, J. (1996). El productor cinematográfico. Madrid: Síntesis. 
Martínez, M. (2003). Las comisiones fílmicas, un nuevo dispositivo para la promoción audiovisual. En Martínez, M. (dir.), Las comisiones fílmicas. Un nuevo dispositivo para la promoción audiovisual. Sevilla: Comunicación social.

Osácar, E. (2009). Del turismo y el cine al turismo cinematográfico. HER\&MUS, 2, pp. 1825.

Palmi, P., Caputo, F. y Turco, M. (2016). Changing Movie! Film Commissions as drivers for creative film industries: the Apulia Case. ENCATC Journal of Cultural Management \& Policy, 6, (1), pp. 56-72.

Pardo, A. (2014). Fundamentos de producción y gestión de proyectos audiovisuales. Barañaín: Eunsa.

Ramahí, D. (2011). Nuevas estrategias comunicativas de ámbito local. El caso de las film commissions u oficinas de atracción de rodajes. En La Publicidad de las instituciones locales. Castelló: Servicio de Publicaciones de Universidad Jaume I.

Rosado, C. y Querol, P. (2006). Cine y turismo. Una nueva estrategia de promoción. Madrid: Ocho y Medio.

Spain Film Comission (2017). Quiénes somos. Recuperado de: http://www.shootinginspain.info/es/quienes-somos 